\title{
URYSON WIDTH AND VOLUME
}

\author{
Panos Papasoglu
}

\begin{abstract}
We give a short proof of a theorem of Guth relating volume of balls and Uryson width. The same approach applies to Hausdorff content implying a recent result of Liokumovich-Lishak-Nabutovsky-Rotman. We show also that for any $C>0$ there is a Riemannian metric $g$ on a 3 -sphere such that $\operatorname{vol}\left(S^{3}, g\right)=1$ and for any map $f: S^{3} \rightarrow \mathbb{R}^{2}$ there is some $x \in \mathbb{R}^{2}$ for which $\operatorname{diam}\left(f^{-1}(x)\right)>C$, answering a question of Guth.
\end{abstract}

\section{Introduction}

The Uryson width is a notion of topological dimension theory that was brought to the realm of Riemannian Geometry by Gromov [Gro83, Gro88, Gro86]. It appears quite naturally in the context of thick-thin decompositions of Riemannian manifolds [Gro96]. Intuitively small $k$-Uryson width means that an $n$-dimensional space 'collapses' to a $k$-dimensional space (where we assume $k<n$ ). For example if we consider a torus $T^{2}=S^{1} \times S^{1}$ where one of the $S^{1}$ 's has very small length $\epsilon$ and the other has, say, length 1 then $T^{2}$ is 'close' (collapses) to the circle of length 1-a lower dimensional manifold. In case that our $n$-dimensional space is non compact, bounded $k$-Uryson width means that the space is 'close' to a $k$-dimensional space.

We recall now the precise definition: if $X$ is a metric space we say that $X$ has $q$-Uryson width $\leq W$ if there exists a $q$-dimensional simplicial complex $Y$ and a continuous map $\pi: X \rightarrow Y$ such that every fiber $\pi^{-1}(y)$ has diameter $\leq W$. We write then that $U W_{q}(X) \leq W$.

Guth [Gut11, Gut17] proved the following theorem answering a conjecture of Gromov:

Theorem 1.1. There exists $\epsilon_{n}>0$ so that the following holds. If $\left(M^{n}, g\right)$ is a closed Riemannian manifold and there exists a radius $R$ such that every ball of radius $R$ in $\left(M^{n}, g\right)$ has volume at most $\epsilon_{n} R^{n}$ then $U W_{n-1}\left(M^{n}, g\right) \leq R$.

Keywords and phrases: Volume, Uryson width, co-area inequality, Hausdorff content

Mathematics Subject Classification: 53C23 
Guth conjectured something stronger that applies to general metric spaces and uses Hausdorff content instead of volume. This was shown recently by LiokumovichLishak-Nabutovsky-Rotman [LLNR19].

The proofs of all these results are somewhat technical as they associate to the space some nice coverings and then approximate the space by the rectangular nerve of these coverings (a method introduced by Gromov [Gro82] and applied in [Gro83], p. 130 to the case of manifolds with a lower Ricci curvature bound). They also use various generalizations of the isoperimetric inequality.

Our aim in this paper is to give a direct proof relying only on the co-area inequality. Our method gives also a new weaker sufficient condition for a space to have small Uryson width. Guth in [Gut17] discusses the relationship between classical topological dimension theory and the quantitative version of this theory for manifolds. In this spirit we show the following quantitative characterization of small Uryson width that resembles the inductive definition of classical topological dimension.

Theorem 3.3. There exist $\epsilon_{n}>0$ so that the following holds. Suppose $X$ is a proper metric space and there exists a radius $R$ such that every ball $B(x, R)$ in $X$ is contained in an open set $U$ with the properties:

1. $U \subseteq B(x, 10 R)$.

2. $H \bar{C}_{n-1}(\partial U) \leq \epsilon_{n} R^{n-1}$.

Then $U W_{n-1}(X) \leq R$.

We denote above by $H C_{n}$ the $n$-dimensional Hausdorff content of a metric spacefor the definition see Sect. 2 .

We note also that Nabutovsky [Nab19] used the method of this paper to give better bounds for the dimensional constant in the celebrated systolic inequality of Gromov.

We give now the idea of the Proof of Theorem 1.1: Let's say that we have a thickened plane $P$, so locally the volume growth is much smaller than $r^{3}$. Then we cut $P$ in pieces of small diameter $<D$ by a 'thickened grid' $G$ (we call this a D-separating subset in sec. 2). Using the co-area inequality (see Lemma 2.5) we show that there is a thickened grid that locally has volume growth much smaller than $r^{2}$ so by induction it admits a map $f$ to a 1-dimensional complex $\Sigma$ with small fibers. By adding finite cones to $\Sigma$ we may extend $f$ to the pieces of $P \backslash G$, so to the whole of $P$. It is easy to see that the fibers of this map have small diameter. We note that our approach is reminiscent of the minimal hypersurface method of Schoen-Yau [SY79a, SY79b] which was used also by Guth [Gut10] in a context similar to ours. Indeed our 'thickened' grid has a rough 'minimal area' property and in some cases can indeed be replaced by a smooth hypersurface. One novelty of our approach is that even when we deal with manifolds we need to consider more general spaces like the grid in the above example.

In Sect. 2 we carry out this proof in the more general context of compact metric spaces and in Sect. 3 we deal with the non-compact case. 
These results imply that there is some $C>0$ such that if $\operatorname{vol}\left(S^{3}, g\right)=1$ then there is a map $f: S^{3} \rightarrow \Sigma$ where $\Sigma$ is a 2-complex and the 'fibers' of the map have diameter bounded by $C$. Guth in [Gut10] asks whether $\Sigma$ may be replaced by $\mathbb{R}^{2}$. In Sect. 4 we give examples showing that the answer is negative.

I am grateful to Stephane Sabourau for pointing out mistakes in an earlier version of this paper and making suggestions that improved the exposition, and to Larry Guth for bringing to my attention the relationship of this approach to minimal surfaces. I thank the referees for their constructive comments that made this paper more readable.

\section{Uryson Width of Compact Metric Spaces}

We prove in this section the generalization of Theorem 1.1 for metric spaces conjectured by Guth. There are some technicalities in the proof as we work with Hausdorff content which is not a measure. We explain in the end how can one give a simpler proof in the manifold case using Hausdorff measure (see Remark 1).

Definition. The $n$-dimensional Hausdorff content $H C_{n}(U)$ of a subset of a metric space $X$ is the infimum of $\sum_{i=1}^{\infty} r_{i}^{n}$ over all coverings of $U$ by countably many balls $B\left(x_{i}, r_{i}\right)$.

We will need a slight variation of Hausdorff content-this will allow us to sidestep the problem that Hausdorff content is not a measure so it is not additive:

Definition. The $\zeta$-restricted $n$-dimensional Hausdorff content $H C_{n}^{\zeta}(U)$ of a subset of a metric space $X$ is the infimum of $\sum_{i=1}^{\infty} r_{i}^{n}$ over all coverings of $U$ by countably many balls $B\left(x_{i}, r_{i}\right)$ where $r_{i} \leq \zeta$ for all $i$.

Clearly we have $H C_{n}^{\zeta}(U) \geq H C_{n}(U)$. We remark that if $U$ is contained in a ball of radius $\zeta$ then $H C_{n}^{\zeta}(U)=H C_{n}(U)$.

Notation. We denote by $B(x, r)$ the open metric ball of radius $r$ and center $x$ and by $\bar{B}(x, r)$ the closed ball. When we don't care about the center we denote it by $B(r)$ $(\bar{B}(r)$ respectively). We denote by $S(x, r)$ the sphere of radius $r$ and center $x$, and we denote this by $S_{r}$ when the center is obvious. Finally we denote by $B\left(r_{2}\right) \backslash B\left(r_{1}\right)$ the annulus between two concentric metric balls.

The co-area formula [BZ13, Theorem 13.4.2] will be our main tool. As we will work in the context of metric spaces it will be crucial below that there is a co-area inequality that applies to Hausdorff content as was shown recently in [LLNR19]. We state this here for $\zeta$-restricted Hausdorff content.

LEMMA 2.1 (Lemma 5.3 of [LLNR19]). Let $U \subset B\left(r_{2}\right) \backslash B\left(r_{1}\right)$ be a closed set of a proper metric space. Then

$$
\int_{r_{1}}^{r_{2}} H C_{n-1}^{\zeta}\left(S_{r} \cap U\right) d r \leq 2 H C_{n}^{\zeta}(U)
$$


where the integral is the Upper Lebesgue integral. The same inequality applies to the Hausdorff content.

Proof. We outline the proof of this from [LLNR19] for the reader's convenience. If $B(R)$ is a ball and $S_{r}$ is a sphere then $S_{r} \cap B(R)$ is contained in a ball of radius $\leq R$ for any $r$, so $H C_{n-1}\left(S_{r} \cap B(R)\right) \leq R^{n-1}$ for any $r$. So if $B(R)$ is a ball contained in an annulus $B\left(r_{2}\right) \backslash B\left(r_{1}\right)$ and $\zeta \geq R$ we have

$$
\int_{r_{1}}^{r_{2}} H C_{n-1}^{\zeta}\left(S_{r} \cap B(R)\right) d r \leq 2 R \cdot R^{n-1} \quad(*) .
$$

Note now that if $U$ is any closed set for any $\epsilon>0$ there is a covering of $U$ by finitely many balls $B_{i}\left(r_{i}\right), i=1, \ldots, k$ so that $r_{i} \leq \zeta$ and $\sum_{i=1}^{k} r_{i}^{n}-H C_{n}^{\zeta}(U)<\epsilon$ and the result follows by $(*)$. Clearly this proof applies to $H C_{n}(U)$ as well.

Guth conjectured in [Gut17] that if a compact (or even proper) metric space has locally small $n$-Hausdorff content then it has small Uryson width. We treat now the easier case $n=1$.

Lemma 2.2. Let $X$ be a proper metric space and let $R>0$. If for any $x \in X$ the 1-dimensional Hausdorff content of the ball $B(x, R)$ is bounded by $\frac{1}{100} R$, then $U W_{0}(X) \leq R$.

Proof. We set $\delta=\frac{1}{100} R$. We fix $x_{0} \in X$ and we consider the closed annuli $A_{k}=$ $\left\{x \in X: 10(k-1) R \leq d\left(x_{0}, x\right) \leq 10 k R\right\}, k \geq 1, k \in \mathbb{N}$. Each $A_{k}$ is compact so it has a finite covering by balls $B_{j}\left(r_{j}\right)$ such that $r_{j} \leq 2 \delta$ for all $j$. Let

$$
a_{k}=H C_{1}^{2 \delta}\left(A_{k}\right) \text {. }
$$

We pick for each $A_{k}$ a covering by open balls $B_{j}\left(r_{j}\right)$ such that

$$
\sum r_{j}-a_{k}<\delta \quad(*)
$$

By doing this for all $k$ we obtain a covering $\mathcal{U}$ of $X$ by open balls.

Suppose that we have a finite sequence of balls in $\mathcal{U}, B_{1}\left(r_{1}\right), \ldots, B_{n}\left(r_{n}\right)$ such that $B_{i}\left(r_{i}\right)$ intersects $B_{i+1}\left(r_{i+1}\right)$ for all $i$. We claim that if this happens then

$$
\sum_{i=1}^{n} r_{i} \leq 10 \delta
$$

We may assume by taking a smaller $n$ if necessary and arguing by contradiction that

$$
12 \delta \geq \sum_{i=1}^{n} r_{i}>10 \delta
$$


So all these balls are contained in a ball $B(x, R)$ which is contained either in a single annulus $A_{k}$ or in a union of two annuli $A_{k} \cup A_{k+1}$. However by our hypothesis the content of $B(x, R)$ is bounded by $\delta$, so we could replace these balls in $\mathcal{U}$ by finitely many balls $B_{s}\left(r_{s}\right), s \in S$ such that their union contains $B(x, R)$ and

$$
\sum_{s \in S} r_{s}<2 \delta
$$

It follows that the sequence $B_{1}\left(r_{1}\right), \ldots, B_{n}\left(r_{n}\right)$ violates $(*)$ for at least one of $A_{k}, A_{k+1}$.

Let $B \in \mathcal{U}$. We note now that if $B_{1}\left(r_{1}\right), \ldots, B_{n}\left(r_{n}\right)$ is a finite sequence of balls from $\mathcal{U}$ containing $B$ such that $B_{i}\left(r_{i}\right)$ intersects $B_{i+1}\left(r_{i+1}\right)$ their union has diameter $<R / 2$.

We introduce an equivalence relation on $\mathcal{U}$. We say that two balls $B, B^{\prime}$ in $\mathcal{U}$ are equivalent if there is a finite sequence of balls $B_{1}=B, B_{2}, \ldots, B_{n}=B^{\prime}$ such that any two successive balls in the sequence intersect.

We replace then each equivalence class of balls from $\mathcal{U}$ by their union.

In this way we obtain a cover of $X$ by sets say $D_{i}, i \in \mathbb{N}$ such that each $D_{i}$ is open (as a finite union of open balls), and closed (since its complement is open). It follows that the map $f: X \rightarrow \mathbb{N}$ where $f\left(D_{k}\right)=k$ is continuous and

$$
\operatorname{diam} f^{-1}(k)=\operatorname{diam} D_{k}<R
$$

so $U W_{0}(X) \leq R$.

If $U$ is an open subset of a Riemannian manifold then $\operatorname{vol}_{n}(U)$ is equal to the $n$-Hausdorff measure of $U$ which is in turn greater or equal to the $n$-dimensional Hausdorff content. It follows that Theorem 1.1 is a corollary of the theorem that we state now-which was conjectured by Guth and proven recently by LiokumovichLishak-Nabutovsky-Rotman [LLNR19]:

Theorem 2.3. There is an $\epsilon_{n}>0$ such that the following holds. If $X$ is a compact metric space such that for any $x \in X$ the $n$-dimensional Hausdorff content of the ball $B(x, R)$ is bounded by $\epsilon_{n} R^{n}$, then $U W_{n-1}(X) \leq R$.

Proof. We will prove by induction on $n$ that there is a continuous map $\pi: X \rightarrow \Sigma$ where $\Sigma$ is a finite simplicial complex of dimension $\leq n-1$ such that $\operatorname{diam} \pi^{-1}(y) \leq R$ for any $y \in \Sigma$. The theorem holds for $n=1$ by Lemma 2.2.

Definition. Let $Z \subseteq X$ closed. We say that $Z$ is a $D$-separating subset if

$$
X \backslash Z=\bigsqcup_{i \in I} U_{i}
$$

where the $U_{i}$ are open disjoint sets of diameter $\leq D$ and $I$ is finite. We say that the open sets $U_{i}$ are the pieces of the decomposition of $X$ by $Z$. 
We set $\zeta=R / 1000$. Let $b(D)$ be the infimum of $H C_{n-1}^{\zeta}(Z)$ over all $D$-separating sets $Z$. It is not clear whether there exists a $D$-separating set realizing $b(D)$ however it will be sufficient for us to consider sets with content close enough to $b(D)$ : We say that $Z$ is a $\delta$-minimal $D$-separating set if $Z$ is $D$-separating and

$$
H C_{n-1}^{\zeta}(Z)-b(D) \leq \delta
$$

In what follows our statements will be true for $\delta$ sufficiently small.

The theorem follows from the next lemma:

Lemma 2.4. There is an $\epsilon_{n}>0$ such that the following holds. If $X$ is a compact metric space such that for any $x \in X$ the $n$-dimensional Hausdorff content of the ball $B(x, R)$ is bounded by $\epsilon_{n} R^{n}$, then there is a finite simplicial complex $\Sigma$ of dimension $\leq n-1$ and a continuous map $f: X \rightarrow \Sigma$ such that: $\operatorname{diam} f^{-1}(e) \leq R$ for any simplex $e \in \Sigma$.

Proof. We prove this by induction on $n$. For $n=1$ the statement follows by Lemma 2.2. In particular we may take $\epsilon_{1}=1 / 100$.

We will show that the lemma holds for $\epsilon_{n}$ where we define $\epsilon_{n}$ inductively by $\epsilon_{n}=\epsilon_{n-1} / 1000^{n+1}$.

We assume now that the lemma holds for $n-1$ for some $n \geq 2$.

Lemma 2.5. Let $\epsilon_{n-1}$ be the constant provided by Lemma 2.4 and let $\epsilon_{n}=$ $\epsilon_{n-1} / 1000^{n+1}$. Let $X$ be a compact metric space such that for any $x \in X$ the $n$-dimensional Hausdorff content of the ball $B(x, R)$ is bounded by $\epsilon_{n} R^{n}$. Let $Z$ be a $\delta$-minimal $R / 4$-separating subset of $X$. Then for any ball of radius $R / 1000$, $B(x, R / 1000)$,

$$
H C_{n-1}^{\zeta}(Z \cap B(x, R / 1000)) \leq \epsilon_{n-1}\left(\frac{R}{1000}\right)^{n-1}
$$

Proof. We argue by contradiction assuming that $Z$ does not satisfy this inequality for some $x$. We take

We note that $(R / 1000)^{n} \geq \epsilon_{n} R^{n}$. It follows that $H C_{n}(B(x, R))=H C_{n}^{\zeta}(B(x, R))$. By the co-area inequality (Lemma 2.1) and our hypothesis that $H C_{n}^{\zeta}(B(x, R)) \leq$ $\epsilon_{n} R^{n}$ we have that for some $r \in[R / 100, R / 50]$

$$
H C_{n-1}^{\zeta} S(x, r) \leq 200 \epsilon_{n} R^{n-1} \leq \frac{\epsilon_{n-1} R^{n-1}}{5 \cdot 1000^{n}}
$$

If $Z_{1}=S(x, r)$ and $Z_{2}=B(x, r) \cap Z$ we set $Z^{\prime}=\left(Z \backslash Z_{2}\right) \cup Z_{1}$. We claim that $Z^{\prime}$ is $R / 4$-separating. Indeed let

$$
X \backslash Z=\bigsqcup_{i \in I} U_{i}
$$


where $I$ is finite and the $U_{i}$ are open disjoint sets of diameter $\leq R / 4$. Let $U=B(x, r)$. Then

$$
X \backslash Z^{\prime}=\bigsqcup_{i \in I}\left(U_{i} \backslash \bar{B}(x, r)\right) \sqcup U
$$

If $B_{i}\left(r_{i}\right), i \in I$ is a cover of $Z$ by balls of radius $\leq \zeta$ so that

$$
\sum_{i \in I} r_{i}^{n-1}-H C_{n-1}^{\zeta}(Z)<\delta
$$

we get a cover of $Z^{\prime}$ by omitting all balls from this cover intersecting $B(x, R / 1000)$ and adding appropriately balls that cover $S(x, r)$ and approximate $H C_{n-1}^{\zeta} S(x, r)$ up to $\delta$.

We have then

$$
H C_{n-1}^{\zeta}\left(Z^{\prime}\right) \leq H C_{n-1}^{\zeta}(Z)-\epsilon_{n-1}\left(\frac{R}{1000}\right)^{n-1}+\frac{\epsilon_{n-1} R^{n-1}}{5 \cdot 1000^{n}}+\delta
$$

contradicting the $\delta$-minimality property of $Z$ if we take

$$
\delta<\frac{\epsilon_{n-1} R^{n-1}}{1000^{n}} .
$$

We prove now Lemma 2.4. Let $Z$ be a $\delta$-minimal $R / 4$-separating subset of $X$. By Lemma 2.5 and our inductive hypothesis there is a continuous map $\pi_{1}: Z \rightarrow \Sigma_{1}$ where $\Sigma_{1}$ is a finite simplicial complex of dimension $\leq n-2$ such that $\operatorname{diam} \pi_{1}^{-1}(e) \leq$ $R / 1000$ for any simplex $e \in \Sigma_{1}$.

Let $U$ be a piece of the decomposition of $X$ by $Z$. Clearly $\partial U \subset Z$ so $\pi_{1}(\partial U)$ is contained in a finite subcomplex of $\Sigma_{1}$. We denote by $\Sigma_{U}$ the minimal such subcomplex of $\Sigma_{1}$.

We define a new simplicial complex $\Sigma$ as follows: For each closure of a connected component $U$ we consider the cone $C_{U}$ over $\Sigma_{U}$ (which is a simplicial complex of dimension $\leq n-1)$. We glue $C_{U}$ to $\Sigma_{1}$ along their common subcomplex $\Sigma_{U}$.

We will need some facts from topology that we recall now (see eg [Hu65]). Any finite simplicial complex is an Absolute Neighborhood Retract (ANR). A contractible ANR is an Absolute Retract (AR). In particular the cone of a finite simplicial complex is an AR. A space $A$ is an AR if and only if it is an absolute extensor i.e. if it has the following property: if $B$ is any metric space, $K \subseteq B$ is closed and $f: K \rightarrow A$ is continuous then $f$ can be extended continuously to the whole of $B$.

By the above facts it follows that for each $U$ the map $\pi_{1}: \partial U \rightarrow \Sigma_{U} \subset C_{U}$ can be extended to a continuous map $\pi: U \rightarrow C_{U} \subset \Sigma$. Since $X$ is the union of $Z$ with the pieces of the decomposition of $X$ by $Z$ and since the map $\pi$ is continuous on the closure of each piece we have that the map $\pi: X \rightarrow \Sigma$ is continuous. 
Let $e$ be a maximal simplex of $\Sigma$. Then $e$ is either a simplex of $\Sigma_{1}$ or a cone of a simplex $e^{\prime}$ of $\Sigma_{1}$. If $\pi(U)$ intersects $e$ then in the first case $\partial U$ intersects $\pi_{1}^{-1}(e)$ while in the second case $\partial U$ intersects $\pi_{1}^{-1}\left(e^{\prime}\right)$. Since

$$
\operatorname{diam} \pi_{1}^{-1}\left(e^{\prime}\right) \leq R / 1000 \text { and } \operatorname{diam}(U) \leq R / 4
$$

we have that

$$
\operatorname{diam} \pi^{-1}(e) \leq R .
$$

We note that an extension argument similar to the one given above appears in section 6.1 of [LLNR19].

Clearly the theorem follows from the lemma as any point of $\Sigma$ is contained in some simplex $e$ of $\Sigma$.

REMARK 1. In the manifold case one could use Hausdorff measure instead of Hausdorff content to prove Theorem 1.1. This would simplify a bit the proof, in particular the Proof of Lemma 2.5. We note however that the subset $Z$ that 'cuts' the space in small pieces that we introduce is not a manifold. So for the proof to work one needs a version of the co-area inequality that applies to spaces with finite Hausdorff measure. We observe that such an inequality follows from Lemma 2.1 by taking a limit as the diameter of the balls approaches 0 (see also [HL86] 1.11, p. 15 or [Fed14] $3.2 .11)$.

\section{The General Case and a Refinement}

We recall that a metric space is called proper if any closed ball is compact. Theorem 2.3 holds more generally for proper metric spaces rather than compact ones. We state here the corresponding inductive statement and explain the modifications needed to prove this.

Theorem 3.1. There is an $\epsilon_{n}>0$ such that the following holds. If $X$ is a proper metric space such that for any $x \in X$ the $n$-dimensional Hausdorff content of the ball $B(x, R)$ is bounded by $\epsilon_{n} R^{n}$, then there is a locally finite simplicial complex $\Sigma$ of dimension $\leq n-1$ and a continuous map $f: X \rightarrow \Sigma$ such that: $\operatorname{diam} f^{-1}(e) \leq R$ for any simplex $e \in \Sigma$. In particular $U W_{n-1}(X) \leq R$.

Proof. The proof is as before by induction on $n$. For $n=1$ the statement follows by Lemma 2.2. We generalize slightly the definition of $D$-separating subset:

Definition. Let $Z \subseteq X$ closed. We say that $Z$ is a $D$-separating subset if

$$
X \backslash Z=\bigsqcup_{i \in I} U_{i}
$$

where the $U_{i}$ are open disjoint sets of diameter $\leq D$ and any ball $B(x, r)$ intersects finitely many of the $U_{i}$ 's. We say that the open sets $U_{i}$ are the pieces of the decomposition of $X$ by $Z$. 
To do the inductive step we fix $x_{0} \in X$ and let

$$
\begin{aligned}
& A_{n}=\left\{x \in X: 10(n-1) R \leq d\left(x, x_{0}\right) \leq 10 n R\right\}, \\
& B_{n}=\left\{x \in X: 10(n-1) R+5 R \leq d\left(x, x_{0}\right) \leq 10 n R+5 R\right\}
\end{aligned}
$$

$(n \geq 1)$. We set $\zeta=R / 1000$. Each $A_{n}, B_{n}$ is compact so we may apply Lemma 2.3. We modify slightly Lemma 2.5: we pick a smaller $\epsilon_{n}$, say,

$$
\epsilon_{n} \leq \frac{\epsilon_{n-1}}{10 \cdot 1000^{n+1}}
$$

and we obtain the following slightly stronger conclusion by the same proof:

Lemma 3.2. Let $Z_{n}$ be a $\delta$-minimal $R / 4$-separating set of $A_{n}$. Then for any ball of radius $R / 1000, B(x, R / 1000)$ of $A_{n}$,

$$
H C_{n-1}^{\zeta}\left(Z_{n} \cap B(x, R / 1000)\right) \leq \frac{1}{10} \epsilon_{n-1}\left(\frac{R}{1000}\right)^{n-1} .
$$

The same lemma applies of course for $\delta$-minimal $R / 4$-separating sets of $B_{n}$ which we denote by $T_{n}$.

Then if

$$
Z=\bigcup_{n=1}^{\infty} Z_{n}, \quad T=\bigcup_{n=1}^{\infty} T_{n}
$$

we claim that $Z \cup T$ is an $R / 4$-separating set of $X$. Indeed if

$$
U_{n}^{i}, \quad V_{n}^{j}, \quad i \in I_{n}, \quad j \in J_{n}
$$

are the pieces of the decomposition of $A_{n}$ by $Z_{n}$, respectively $B_{n}$ by $T_{n}$ we set

$$
I=\bigcup_{n=1}^{\infty} I_{n}, J=\bigcup_{n=1}^{\infty} J_{n}
$$

and

$$
I^{\prime}=\left\{i \in I: U_{i} \text { is open in } \mathrm{X}\right\}, J^{\prime}=\left\{j \in J: V_{j} \text { is open in } \mathrm{X}\right\} .
$$

Then we may take the open sets

$$
W_{i j}=U_{i} \cap V_{j}, \quad i \in I^{\prime}, j \in J^{\prime}
$$

to be the pieces of the decomposition of $X$ by $Z \cup T$. Clearly $\bigcup W_{i j}=X \backslash(Z \cup T)$. Each ball intersects finitely many of these sets since it intersects finitely many of $U_{i}, V_{j}$. Applying Lemma 3.2 we see that $Z \cup T$ satisfies the hypothesis of Theorem 3.1 for $n-1$ and the same proof as in Theorem 2.4 applies in this case too.

Our method allows us to give a weaker sufficient condition for a space to have small Uryson width. We remark that the volume condition given in Theorem 2.3 for a space to have small Uryson width is not a necessary one. For example one can 
have a ball $B$ of radius $R$ with $H C_{n}(B)=R^{n}$ and $U W_{1}(B)<\epsilon$ for arbitrarily small $\epsilon$. Recall that a metric space $X$ has inductive dimension $\leq n$ if every point $x \in X$ has arbitrarily small neighborhoods $U$ such that $\operatorname{dim} \partial U \leq n-1$ (see [HW69], def. III.1, p. 24). It turns out that for separable metric spaces the inductive dimension is equal to the covering (known also as topological) dimension (see [HW69], thm V 8, p. 67).

We give a quantitative statement similar to the definition of inductive dimension where we assume for $\partial U$ small $n-1$-Hausdorff content rather than dimension. So this is a result in the spirit of passing from a qualititative theorem from topological dimension theory into some quantitative estimates (for more on this theme see [Gut17], sec. 0.2).

Theorem 3.3. There exist $\epsilon_{n}>0$ so that the following holds. Suppose $X$ is a proper metric space and there exists a radius $R$ such that every ball $B(x, R)$ in $X$ is contained in an open set $U$ with the properties:

1. $U \subseteq B(x, 10 R)$.

2. $H \bar{C}_{n-1}(\partial U) \leq \epsilon_{n} R^{n-1}$.

Then $U W_{n-1}(X) \leq R$.

Proof. One argues as in the Proof of Theorem 3.1. Here we don't need the co-area formula as we assume that $H C_{n-1}(\partial U)$ is small. So we can prove as in Lemma 2.5 that a $\delta$-minimal $1000 R$ separating subset $Z$ has locally small $H C_{n-1}^{\zeta}$ and the rest of the proof is identical.

We remark however that this theorem does not provide a characterization of spaces $X$ with small $U W_{n-1}(X)$. For example consider a 3 -regular metric tree $T$ where each edge has length $\delta>0$. Let $X=T \times[0, \epsilon]$. Clearly $U W_{1}(X) \leq \epsilon$ but by picking $\delta$ very small we see that $H C_{1}(\partial U) \geq R$ for open sets $U$ containing an $R$-ball.

\section{An Example}

Balacheff-Sabourau showed in [BS10] that there is a constant $c_{g}$ such that if $S_{g}$ is a Riemannian surface of genus $g$ and volume 1 then there is a map $f: S_{g} \rightarrow \mathbb{R}$ such that the length of the level sets $f^{-1}(x)$ is bounded by $c_{g}$. Guth in [Gut10], p. 765 asks if there is a similar bound for maps from the 3 -sphere to $\mathbb{R}^{2}$ where we assume again that vol $S^{3}=1$. In this section we show that for any $C>0$ there is a Riemannian metric $g$ on the 3 -sphere with $\operatorname{vol}\left(S^{3}, g\right)=1$ such that for any map $f: S^{3} \rightarrow \mathbb{R}^{2}$ there is some $x \in \mathbb{R}^{2}$ for which $\operatorname{diam}\left(f^{-1}(x)\right)>C$ answering this question in the negative. We note that Theorem 1.1 implies that there is $C>0$ such that for any Riemannian metric $g\left(S^{3}, g\right)$ of volume 1 there a map $f: S^{3} \rightarrow \Sigma$ for some 2-complex $\Sigma$ such that for any $x \in \Sigma \operatorname{diam}\left(f^{-1}(x)\right) \leq C$. So the example really shows that we can not replace $\Sigma$ by $\mathbb{R}^{2}$. It remains open whether one can find 
a map $f: S^{3} \rightarrow \Sigma$ where $\Sigma$ is a 2-complex so that the length of the level sets (rather than the diameter) is bounded (see [Gut10]).

One could see maps $f: M \rightarrow \mathbb{R}^{n}$ where $M$ is a manifold as 'higher analogs' of Morse functions-especially if one imposes some smoothness conditions on $f$. Such maps were studied extensively in [Gro09] and in [Gro10].

Our construction is based on a simple observation about graphs. Namely the fact that the non-planarity of the complete graph $K_{5}$ has a quantitative version: if we take a copy of $K_{5}$ where the edges are very long then in any drawing of this graph on the plane two 'far away' points get identified. We give a formal proof of this in the next two lemmas.

Let $\Gamma=(V, E)$ be a graph. We consider here metric graphs, that is edges are isometric to some interval $[0, \ell]$ and the distance between two points is the length of the shortest path joining them.

It will be convenient to identify some planar subsets with graphs: We will say that a subset of the plane $\mathbb{R}^{2}$ is a graph if it is a finite union of closed arcs and any two arcs may intersect only at their endpoints. We say that these arcs are the edges of the graph. We call the subset of the plane consisting of endpoints of these arcs the set of vertices of the graph. We note that a subset of the plane can have more than one graph structure as one can always subdivide some edges and add vertices. So when we talk of a graph on the plane we assume that we have fixed a choice of vertices and edges.

We recall a classical topological lemma (see Corollary 31.6 in [Wil04]).

Lemma 4.1. Let $X$ be a metric space and let $f:[0,1] \rightarrow X$ be a continuous path joining $x, y$. Then there is a simple path joining $x, y$ contained in $f([0,1])$.

LemMA 4.2. Let $K_{5}=(V, E)$ be the complete graph with 5 vertices metrized so that each edge has length $10 R$. If $f: K_{5} \rightarrow \mathbb{R}^{2}$ is continuous, then there is some $x \in \mathbb{R}^{2}$ with $\operatorname{diam} f^{-1}(x)>R$.

Proof. We argue by contradiction, that is we assume that $\operatorname{diam} f^{-1}(x) \leq R$ for all $x$. For any vertex $v \in V$ we consider the ball of radius $2 R, B(v, 2 R)$ in $K_{5}$. This is a tree $T$ consisting of a union of intervals $\left[v, x_{i}\right], i=1,2,3,4$ intersecting only at $v$. By Lemma 4.1 there is a simple arc $\alpha_{i}$ with endpoint $f(v), f\left(x_{i}\right)$ contained in $f\left(\left[v, x_{i}\right]\right)$. Let $p_{1}$ be the last intersection point on $\alpha_{2}$ between $\alpha_{1}, \alpha_{2}$. We replace then $\alpha_{2}$ by the subarc of $\alpha_{2}$ with endpoints $p_{1}, f\left(x_{2}\right)$. Call this arc $\beta_{2}$ Similarly we consider the last intersection point $p_{2}$ of $\alpha_{3}$ with $\alpha_{1} \cup \beta_{2}$ and we replace $\alpha_{3}$ by its subarc of $\alpha_{3}$ with endpoints $p_{2}, f\left(v_{3}\right)$. We repeat this with the other arcs and we note that $\alpha_{1} \cup \beta_{2} \cup \cdots \cup \beta_{4}$ is a tree with 4 (or 5) endpoints contained in $f(T)$. We do this for all 5 vertices of $K_{5}$ and we obtain 5 trees on the plane $T_{i}, i=1, \ldots, 5$. By Lemma 4.1 for each pair $T_{i}, T_{j}$ there is a simple arc $\alpha_{i j}$ joining an endpoint of $T_{i}$ with an endpoint of $T_{j}$. We note that $\alpha_{i j}$ might intersect $T_{i}, T_{j}$ at many points. We replace $\alpha_{i j}$ by a subarc $\beta_{i j}$ such that $\beta_{i j}$ intersects $T_{i} \cup T_{j}$ only at its endpoints and one endpoint is in $T_{i}$ and another is in $T_{j}$. By our hypothesis the arcs $\beta_{i j}$ do 
not intersect each other and the trees $T_{i}$ do not intersect either. So the union of the $T_{i}$ 's with the $\beta_{i j}$ 's is a planar graph. However it is clear that this graph has $K_{5}$ as a minor-just contract the trees $T_{i}$ to points. This is clearly a contradiction since $K_{5}$ is not planar.

The construction. The idea is that we may construct a 3 -sphere with volume 1 that contains an almost isometric copy of $K_{5}$ where the edges of $K_{5}$ are very long. The point is we can replace $K_{5}$ with a very thin handlebody $\Sigma$ and $S^{3}$ can be obtained by gluing two such thin handlebodies. To arrange that $K_{5}$ is almost isometrically embedded we interpolate $\Sigma \times[0, L]$ for some big $L$ between the two copies. We explain now this more formally.

Given $C>0$ pick a graph $\Gamma$ isomorphic to $K_{5}$ so that the edges of $\Gamma$ have length $100 C$. Thicken $\Gamma$ so that is becomes a handlebody $\Sigma$. We may choose a Riemannian metric on $\Sigma$ so that $\Sigma$ is contained in an $\epsilon$ neighborhood of $\Gamma$, its volume is less than $\epsilon$, its boundary surface $S$ and $\Sigma$ are both $(1, \epsilon)$ quasi-isometric to $\Gamma$ and $\operatorname{area}(S)<\epsilon$, where $\epsilon$ is a small positive number that we will specify later. Glue to $\Sigma$ along $S$ the manifold $S \times[0,100 C]$ with the product metric to obtain a handlebody $M$. Clearly $\operatorname{vol}(M)<(100 C+1) \epsilon$. Consider now a small copy $S_{1}$ of $S$ of diameter $<1 / 10$ embedded in the standard sphere $S^{3}$ of volume $1-\delta$ where $\delta$ is a small positive number to specify later. Denote the handlebody of diameter $<1 / 10$ bounded by $S_{1}$ in $S^{3}$ by $\Sigma_{1}$. Consider now a Riemannian metric $g_{1}$ on $S \times[0,1]$ interpolating between the metric on $\partial M$ and $S_{1}$, so $S \times\{0\}$ is isometric to $S$ and $S \times\{1\}$ is isometric to $S_{1}$. We may further assume that the volume of $\left(S \times[0,1], g_{1}\right)$ is smaller than $2 \epsilon$. We glue now $S \times\{0\}$ to $M$ and $\partial M$ and $S \times\{1\}$ to $S^{3} \backslash S_{1}$ by isometries. By appropriately picking $\delta, \epsilon$ and smoothing the metric we obtain a Riemannian sphere $\left(S^{3}, g\right)$ with $\operatorname{vol}\left(S^{3}, g\right)=1$. Since $\Gamma$ is $(1, \epsilon)$ quasi-isometrically embedded in this sphere clearly Lemma 4.2 implies that for any $f: S^{3} \rightarrow \mathbb{R}^{2}$ there are $x, y \in \Gamma$ with $d(x, y)>C$ and $f(x)=f(y)$.

We note that this construction is very similar to a construction in proposition 0.10 in [Gut17] which is turn is similar to example $\left(H_{1}^{\prime \prime}\right)$ of [Gro88].

Larry Guth after seeing this example suggested another way to obtain such metrics: start with a tripod $T$ with long edges. Clearly any map from the tripod to $\mathbb{R}$ has some fiber of long diameter. Consider $X=T \times[0, L]$ for some big $L$. Again any map $f: X \rightarrow \mathbb{R}^{2}$ has some big fiber. Now thicken $X$ to obtain a 3 -ball and glue two such balls along their boundaries to obtain a 3 -sphere $S$ such that for any $f: S \rightarrow \mathbb{R}^{2}$ there is a point $x \in \mathbb{R}^{2}$ for which diam $f^{-1}(x)$ is large.

Open Access This article is licensed under a Creative Commons Attribution 4.0 International License, which permits use, sharing, adaptation, distribution and reproduction in any medium or format, as long as you give appropriate credit to the original author(s) and the source, provide a link to the Creative Commons licence, and indicate if changes were made. The images or other third party material in this article are included in the article's 
Creative Commons licence, unless indicated otherwise in a credit line to the material. If material is not included in the article's Creative Commons licence and your intended use is not permitted by statutory regulation or exceeds the permitted use, you will need to obtain permission directly from the copyright holder. To view a copy of this licence, visit http://creativecommons.org/licenses/by/4.0/.

Publisher's Note Springer Nature remains neutral with regard to jurisdictional claims in published maps and institutional affiliations.

\section{References}

[BS10] F. BAlachefF and S. SAbourau. Diastolic and isoperimetric inequalities on surfaces. Annales scientifiques de l'École Normale Supérieure, (4)43 (2010), 579605

[BZ13] Y.D. Burago and V.A. Zalgaller. Geometric Inequalities, Vol. 285. Springer, Berlin (2013)

[Fed14] H. Federer. Geometric Measure Theory. Springer, Berlin (2014)

[Gut11] L. Guth. Volumes of balls in large Riemannian manifolds. Annals of Mathematics, (173 )173 (2011), 51-76

[Gut17] L. Guth. Volumes of balls in Riemannian manifolds and Uryson width. Journal of Topology and Analysis, (9)9 (2017), 195-219

[Gut10] L. Guth. Systolic inequalities and minimal hypersurfaces. Geometric and Functional Analysis, (6)19 (2010), 1688-1692.

[Gut10] L. Guth. Metaphors in systolic geometry. In: Proceedings of the International Congress of Mathematicians 2010 (ICM 2010) (In 4 Volumes) Vol. I: Plenary Lectures and Ceremonies Vols. II-IV: Invited Lectures (2010).

[Gro83] M. Gromov. Filling riemannian manifolds, Journal of Differential Geometry, (1)18 (1983), 1-147.

[Gro88] M. Gromov. Width and related invariants of Riemannian manifolds. Asterisque, (164)163 (1988), 93-109.

[Gro86] M. Gromov. Large Riemannian Manifolds. Curvature and Topology of Riemannian Manifolds. Springer, Berlin (1986), pp. 108-121.

[Gro96] M. Gromov. Systoles and intersystolic inequalities. Actes de la Table Ronde de Géométrie Différentielle (Luminy, 1992) Soc. Math. France, 1 (1996), 291-362

[Gro82] M. Gromov. Volume and bounded cohomology. Publications Mathématiques de l'IHÉS, (56)1982 (1983), 5-99

[Gro09] M. Gromov. Singularities, expanders and topology of maps. Part 1: homology versus volume in the spaces of cycles. Geometric and Functional Analysis, (3)19 (2009), 743-841

[Gro10] M. Gromov. Singularities, expanders and topology of maps. Part 2: from combinatorics to topology via algebraic isoperimetry. Geometric and Functional Analysis, (2)20 (2010), 416-526

[Hu65] S.T. Hu. Theory of Retracts. Wayne State University Press, Detroit (1965).

[HL86] R. HARDT and L. Simon. Seminar on Geometrical Measure Theory. Birkhäuser, Basel (1986).

[HW69] W. Hurewicz and H. Wallman. Dimension Theory, Vol. 4. Princeton university press, Princeton (1969). 
[LLNR19] Y. Liokumovich, B. Lishak, A. Nabutovsky and R. Rotman. Filling metric spaces (2019). arXiv preprint arXiv:1905.06522

[Nab19] A. Nabutovsky. Linear bounds for constants in Gromov's systolic inequality and related results (2019). arXiv preprint arXiv:1909.12225

[SY79a] R. Schoen and S.T. YAU. On the structure of manifolds with positive scalar curvature. Manuscripta Mathematica (1)28 (1979), 159-183

[SY79b] R. Schoen and S.T. YAu. On the proof of the positive mass conjecture in general relativity. Communications in Mathematical Physics (1)65 (1979), 4576.

[Wil04] S. Willard. General Topology. Courier Corporation, London (2004).

Panos Papasoglu, Mathematical Institute, University of Oxford, Andrew Wiles Building, Woodstock Rd, Oxford OX2 6GG, UK.

papazoglou@maths.ox.ac.uk

Received: October 3, 2019

Revised: February 12, 2020

Accepted: February 15, 2020 